ISSN 2693-2504

\title{
Tocilizumab in Severe Covid-19 Infection, Early Experiences at a Community Hospital
}

Journal of Medical Clinical Case Reports

Research Article

Alwyn Rapose ${ }^{1 *}$, Raul Davaro ${ }^{2}$

${ }^{1} M D$, Reliant Medical Group, Division of Infectious Diseases

*Correspondence author

${ }^{2}$ MD, Reliant Medical Group, Division of Infectious Diseases

\author{
Alwyn Rapose, MD. \\ Reliant Medical Group, Division of Infectious \\ Diseases. \\ 123 Summer Street, Suite 220 \\ Worcester, MA. 01608 \\ Submitted : 30 Apr 2021 ; Published : 15 May 2021
}

\begin{abstract}
The COVID-19 global pandemic has resulted in more than 118 million cases and more than 2.5 million deaths. Most fatalities are related to severe pulmonary disease caused by a severe inflammatory response to the virus in the lungs. As yet there is no uniformly successful therapeutic agent. Tocilizumab is a monoclonal antibody that binds to IL-6 receptor thus inhibiting IL-6 mediated signaling of the inflammatory cascade. Early trials did not show a mortality benefit in patients treated with this agent. Newer studies have shown a benefit when Tocilizumab is used in conjunction with corticosteroids. This is a brief report on 7 patients with severe COVID-19 infection who received Tocilizumab therapy at our community hospital in the early period of the pandemic.

Methods

A protocol for the use of Tocilizumab in patients with severe COVID-19 infection was established by the division of Infectious Diseases. Pretreatment laboratory tests included IL-6, ferritin, CRP. Patients received one intravenous infusion of 4 to $8 \mathrm{mg} / \mathrm{kg}$ (maximum dose $800 \mathrm{mg}$ ). The response in 7 consecutive patients who received Tocilizumab was evaluated.

Results

Two out of the 7 patients had good outcomes. Five patients had complicated hospital courses, 2 of them died (mortality 28.5\%). IL-6 levels were elevated at baseline and there was a marked increase following the dose.

Conclusions

Tocilizumab benefitted two previously healthy young male patients with severe COVID-19 infection who received the dose early in the disease. Benefit was not seen in five older individuals with advanced disease. Our experience adds to the accumulating data regarding the limited benefit of single dose Tocilizumab in patients with advanced COVID-19 disease. The dose and optimal time of administration of Tocilizumab, as well as benefits when employed along with other agents like corticosteroids, remdesivir and convalescent plasma needs to be evaluated further.
\end{abstract}

Keywords: COVID-19 pneumonia, Interleukin 6, Tocilizumab

\section{Introduction}

The coronavirus most typically causes mild respiratory illnesses in humans [1]. In 2002 there was an outbreak of severe infections - severe acute respiratory syndrome (SARS), that originated in South China and affected nearly 30 countries. In 2012 there was another outbreak affecting more than 25 countries and it was called Middle East Respiratory Syndrome (MERS) because a majority of the cases were reported from Saudi Arabia. In December 2019 there was an outbreak of coronavirus-related-pneumonia in Wuhan, China which rapidly spread all over the world causing the pandemic we now know as COVID-19. The number of cases worldwide is more than 118 million (2.6 million deaths), with close to 30 million cases reported in the United States where there have been more than 500,000 deaths.
While multisystem disease manifestations have been described, $[2,3]$ the majority of deaths in patients with severe COVID-19 involves respiratory failure [3, 4]. Computed tomography (CT) images of the lungs in these patients reveal extensive bilateral infiltrates, and laboratory tests reveal marked elevation in acute-phase reactants like c-reactive protein (CRP), ferritin, d-dimer and pro-inflammatory cytokines like interleukin-1 and interleukin-6 (IL-6) referred to as the "cytokine storm" $[5,6]$.

Tocilizumab (TCZ) is a monoclonal antibody that binds to the IL-6 receptor thus inhibiting IL-6 mediated signaling of the inflammatory cascade. It is approved for use in rheumatoid arthritis [7]. In the early months of the COVID-19 pandemic, while awaiting results of clinical trials and specific guidelines from the US Center for Disease Prevention and Control 
(CDC), many institutions started using TCZ in patients with severe infection who were failing other treatments. This is a brief report on 7 patients with severe COVID-19 infection who received TCZ therapy at our community hospital in the early period of the pandemic.

\section{Methods}

A hospital-wide protocol for the use of TCZ in patients with severe COVID-19 infection was established by the division of Infectious Diseases. Candidates who had COVID-19 infection confirmed by a positive PCR result, had pulmonary disease confirmed by chest radiography or CT scan, had oxygen saturation less than $93 \%$ or $\mathrm{PaO} 2 / \mathrm{FiO} 2$ less than $300 \mathrm{mmHg}$, those who were mechanically ventilated, and had clinical, radiographic or arterial blood gas evidence of deterioration despite other therapies were eligible to receive TCZ. Exclusion criteria included severe allergic reaction to TCZ or other monoclonal antibodies, active bacterial, fungal or viral (other than COVID-19) infection, patients who had received oral antirejection or immuno-modulator drugs within the past 6 months, pregnancy, breast-feeding, AST or ALT greater than 10 times upper limit of normal, absolute neutrophil count less than 1000 and platelet count less than 50,000. Pretreatment laboratory tests included IL-6, ferritin, CRP. The dosing protocol was for one intravenous infusion of 4 to $8 \mathrm{mg} / \mathrm{kg} \mathrm{TCZ}$ (maximum dose $800 \mathrm{mg}$ ).

\section{Results}

In the period April 1st to April 10th, seven patients - 4 males and 3 females - admitted to the intensive care unit (ICU) with COVID-19 infection received a therapeutic trial with TCZ (see clinical characteristics and baseline inflammatory markers in table 1). Response was evaluated in terms of clinical status (fever, oxygenation) as well as results of inflammatory markers: CRP (normal: 0-5 mg/L), IL-6 (normal: $<2 \mathrm{pg} / \mathrm{mL}$ ), ferritin (normal: $30-400 \mathrm{ng} / \mathrm{mL}$ ), d-dimer (normal: $<0.5 \mathrm{mg} / \mathrm{L}$ ). The median age was 50.5 years (range: 28 to 73 years). Median time for TCZ administration was 7.5 days (range: 2 to 13 days) from the day of admission to ICU.

\section{Case 1}

A 28-year-old male was admitted to the intensive care unit (ICU) on day 4 of hospitalization secondary to severe hypoxia with oxygen saturations $90 \%$ on $50 \%$ venti-mask, and persistent fevers as high as $1030 \mathrm{~F}$. Radiology (chest X-ray as well as CT scan) revealed diffuse bilateral airspace disease. He received TCZ $800 \mathrm{mg}$ on day 2 in the ICU.

Clinical response: He became afebrile the day after (day 2) he received TCZ, and his respiratory status improved, saturating $91 \%$ on high flow nasal cannula, then $95 \%$ on $6 \mathrm{~L}$ nasal cannula on day 4 . He was discharged with home oxygen therapy on day 5.

Laboratory changes: CRP was 239.9 the day prior to TCZ, reduced to 61.5 on day 3. IL-6 was 47 prior to TCZ, increased to 1412 on day 2. Ferritin level was 1569 prior to TCZ, increased to 1783 on day 2, d-dimer was 1.92 prior to TCZ, was not obtained in follow-up.

\section{Case 2}

A 48-year-old male was admitted to the ICU on day 3 of hospitalization secondary to persistent fevers $(101.80 \mathrm{~F})$ and worsening shortness of breath requiring 100\% oxygen via nonrebreather mask. Chest x-ray revealed focal left basilar airspace disease, CT scan of the chest revealed multiple bilateral peripheral patchy areas of consolidation, predominantly in the lower lobes. He received $800 \mathrm{mg}$ of TCZ on day 5 in the ICU.

Clinical response: He became afebrile prior to receiving TCZ, but was persistently tachypneic, hypoxic, saturating $86 \%$ on high flow nasal cannula. After TCZ dose he gradually improved. After 48 hours (day 3) he was saturating 93\% on high flow nasal cannula, he was weaned down to $94 \%$ on $6 \mathrm{~L}$ nasal cannula over the next 48 hours. However he remained tachypneic over the next 2 days, so a repeat CT scan was performed. This revealed persistent infiltrates consistent with COVID-19 pneumonia, in addition he had acute filling defects in the right lower lobe segmental pulmonary branches consistent with pulmonary embolism. He was placed on apixaban twice daily and was discharged home 2 days later. $\mathrm{He}$ did not require oxygen therapy at discharge.

Laboratory changes: CRP was 148 prior to TCZ, came down to 84 on day 3 .

IL-6 was 64.6 prior to TCZ, increased to 517.2 on day 3 . Ferritin level was 1736 prior to TCZ, increased to 2430 on day 3. D-dimer was 0.59 prior to TCZ, was not obtained in followup.

Case 3

A 58-year-old male presented to the emergency room with severe hypoxia requiring endotracheal intubation and admission to the ICU. Chest $\mathrm{x}$-ray on admission revealed bilateral lung infiltrates with relative sparing of the apices. On day 4 he was afebrile, however was in severe respiratory distress with respiratory rate $37 / \mathrm{min}$, saturating $94 \%$ on $100 \%$ FiO2. X-ray of the chest showed progressing airspace disease and he received $600 \mathrm{mg}$ TCZ.

Clinical response: 48 hours after receiving TCZ he remained afebrile, but continued with severe respiratory distress, breathing $38 / \mathrm{min}$ saturating $92 \%$ on $100 \% \mathrm{FiO} 2$. Prone ventilation was attempted. He proceeded to develop multiple complications including ventilator-associated-pneumonia (VAP) with methicillin sensitive staphylococcus aureus (MSSA), persistent respiratory failure requiring tracheostomy, renal failure requiring dialysis and prolonged hospitalization (66 days) after which he was discharged to a rehabilitation facility.

Laboratory changes: CRP was 203.76 on admission, it reduced to 159.1 on day 2 after TCZ dose. IL- 6 was 625.9 on admission, it was not checked post TCZ. Ferritin was 767 prior to TCZ, was not checked following the dose. D-dimer was 17.37 prior to dose and increased to 35.2 on day 3 .

Case 4

A 61-year-old female who was admitted to the hospital secondary to fever and shortness of breath, was transferred to 
the ICU on day 3 secondary to respiratory failure requiring mechanical ventilation. Her chest x-ray revealed bilateral central and peripheral hazy opacities, her chest CT scan showed patchy peripheral ground-glass opacities. She had persistent fever (101.90 F) and persistent hypoxia. On day 10 of her ICU stay she received $600 \mathrm{mg}$ TCZ.

Clinical response: 48 hours after receiving TCZ she became afebrile. However she remained hypoxic on mechanical ventilation, developed acute pneumothorax, and she passed away after a prolonged ICU stay (42 days).

Laboratory changes: Her CRP was 147.4 prior to TCZ, increased to 315 the day after (day 2) receiving TCZ, reduced to 3.09 after 2 weeks. IL-6 level was 53.5 prior to TCZ, it increased to greater than 3000 on day 2 after TCZ dose, remained elevated (1185) for more than 1 week. Ferritin level was 3144 prior to TCZ, it decreased to 1119 after the dose. D-dimer was 5.24 prior to TCZ, it was not checked post TCZ dose.

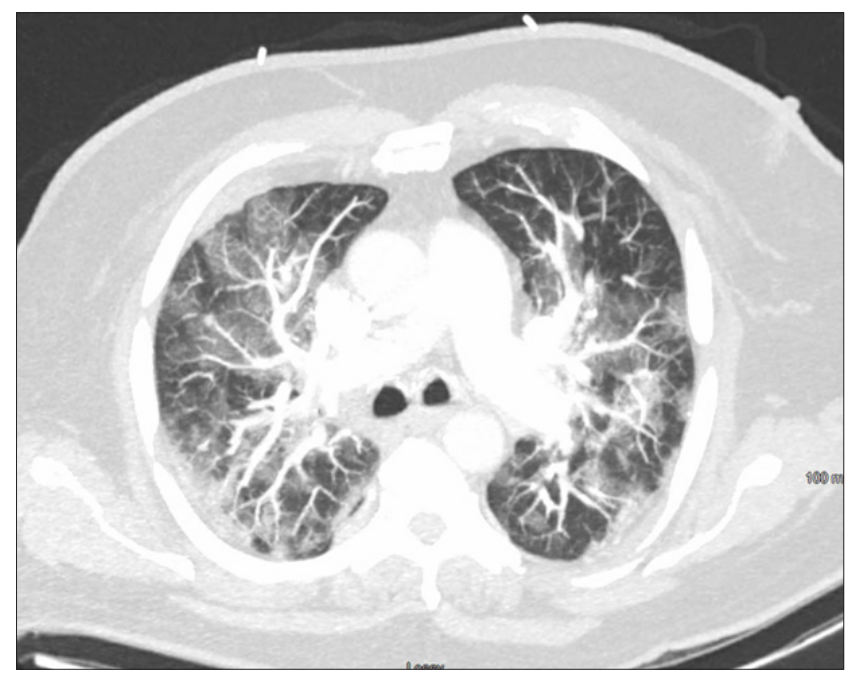

Figure 1: CT scan chest showing diffuse bilateral ground glass pulmonary infiltrates

Case 5

A 73-year-old female admitted to the hospital secondary to severe shortness of breath, cough and fever (103.20 F). She had progressive worsening hypoxia and was transferred to the ICU 5 days after admission to the hospital. Initial chest x-ray had shown no acute cardiopulmonary process, however CT scan of the chest showed predominantly peripheral multifocal ground-glass opacities in bilateral lung fields. In the ICU she was afebrile, but remained hypoxic saturating 95\% on nonrebreather mask and intermittent Bi-PAP ventilation. On her third day in the ICU she received $800 \mathrm{mg}$ TCZ.

Clinical response: Patient's respiratory status continued deteriorating, she required intubation and mechanical ventilation 4 days after dose of TCZ. She proceeded to develop multiple complications including VAP with Klebsiella and Enterobacter, bilateral pleural effusions, persistent respiratory failure requiring tracheostomy, right-sided hemiplegia secondary to stroke and prolonged hospitalization (70 days), after which she was discharged to a rehabilitation facility.
Laboratory changes: CRP was 241.37 prior to TCZ dose, it came down to 118.71 after 48 hours. IL-6 was 238.1 prior to TCZ dose, it went up to greater than 3000 after TCZ. Ferritin level was 624 prior to dose, it went up to 634 on day 3 after the dose. D-dimer was 0.86 prior to TCZ, it was not checked post TCZ dose.

\section{Case 6}

A 61-year-old male was admitted to the ICU with severe shortness of breath requiring endotracheal intubation in the emergency room. His temperature on presentation was 100.20 F. A chest x-ray revealed diffuse multifocal bilateral airspace consolidations. On ICU day 2 he received $400 \mathrm{mg}$ TCZ.

Clinical response: Prior to TCZ patient was afebrile, oxygen saturation was 87 to $90 \%$ on $80 \% \mathrm{FiO} 2$. On day 2 after TCZ dose he was weaned down to $50 \% \mathrm{FiO} 2$. He required prolonged ventilator support and was extubated 11 days after TCZ dose. However, he developed VAP with Acinetobacter and acute renal injury. He was transferred out of the ICU on day 15 and discharged to a rehabilitation facility on day 20 .

Laboratory changes: CRP was 191.25 prior to TCZ dose, went up to 279.4 on day 3 after the dose, then reduced to 59.7 on day 6. IL-6 level was not obtained. Ferritin level was 948 prior to TCZ, it increased to 3039 after the dose. D-dimer was 2.35 prior to dose of TCZ, it was 1.86 on day 7 after TCZ dose.

Case 7

A 57-year-old female admitted to the hospital with fever and severe hypoxia. She was intubated in the emergency room and transferred to the ICU for mechanical ventilation. Chest x-ray revealed multiple hazy opacities in the bilateral lung fields. She had persistent fever (1020 F), also persistent hypoxia saturating $90 \%$ on $70 \% \mathrm{FiO} 2$. She received TCZ $400 \mathrm{mg}$ on day 5 in the ICU.

Clinical response: She became afebrile 5 days after the dose of TCC. However she remained hypoxic on mechanical ventilation, developed VAP with Serratia, and she passed away after a prolonged ICU stay (50 days).

Laboratory changes: CRP, IL-6 and ferritin were 314.20, 2481.3 and 808 respectively prior to the dose of TCZ, these were not checked in the days following the dose. D-dimer was 1.42 prior to the dose of TCZ, it went up to 17.70 on day 4 after the dose.

\section{Summary}

We describe 7 patients with severe COVID-19 infection who received one dose of TCZ. Diffuse bilateral airspace disease was consistently seen in all 7 cases (see representative CT scan image in figure 1). Two out of the 7 patients had good outcomes. Five patients had prolonged complicated hospital course, 4 patients developed superadded bacterial pneumonia and 2 of them died (mortality 28.5\%). IL-6 levels were obtained in 6 out of 7 patients prior to TCZ dose. Values ranged from 47 - 2481.3. Post-TCZ levels were obtained in 4 patients and in all four there was a marked increase in IL-6 level following the dose $(517$ to $>3000)$. Similar increase in ferritin level was noted post-TCZ dose in 3 patients, while it was decreased in 
one patient. CRP decreased in 4 patients, but increased in 2 patients after the dose of TCZ. Follow-up D-dimer post-TCZ dose was available in 3 of the patients and it increased in all 3 patients after dose of TCZ.

\section{Discussion}

Severe COVID-19 infection is characterized by an exacerbated inflammatory response as evidenced by markedly elevated inflammatory markers and interleukins [6]. Since the beginning of the pandemic many different therapies (vitamin D, vitamin C, zinc, H2 blockers, antibiotic: azithromycin, antiviral: lopinavir, antiparasite: ivermectin, convalescent plasma) have been offered to patients. Multiple clinical trials using many different medications are still underway all over the world with conflicting results. TCZ is a recombinant monoclonal antibody directed against IL-6 receptor. Intravenous TCZ 8 $\mathrm{mg} / \mathrm{kg}$ administered every 4 weeks has been shown to be safe and highly effective in the treatment of rheumatoid arthritis, with infections being the most commonly reported adverse event [7]. Around March 26th, 2020, the US-FDA approved the Phase III clinical trial of TCZ in hospitalized patients with severe COVID-19 infection [8].

Our report reviews the results in 7 patients with severe infection early in the COVID-19 pandemic (April 2020) in the United States. The antiviral drug remdesivir was not available at that time. All 7 patients received a therapeutic trial with hydroxychloroquine prior to dose of TCZ. They also received antibiotics empirically on admission (see table 2) and these were de-escalated to target the organisms seen on respiratory cultures. Cultures were available in 5 out of 7 patients prior to dose of TCZ, none of them yielded a pathogen. The patients had markedly elevated IL-6 levels and these increased following the dose of TCZ.

In our small observational study, we noted a benefit in only 2 out of 7 patients who received TCZ therapy. Both were young males less than 50 years of age with no significant comorbidities. Both the patients received TCZ prior to need for endotracheal intubation and mechanical ventilation. Patient 1 had rapid resolution of his shortness of breath and was discharged home 5 days after dose of TCZ. Patient 2 also had relatively good response to TCZ with gradual improvement in oxygen saturation. His course was complicated by pulmonary embolism. He was discharged home 10 days after he received TCZ. The remaining 5 patients had prolonged hospitalizations with multiple complications. Of these, 2 patients died, patient 3 developed MSSA pneumonia, patient 5 developed pneumonia with Klebsiella and Enterobacter, patient 6 developed pneumonia with Acinetobacter Bowmanii, and patient 7 developed Serratia pneumonia. TCZ has been associated with serious adverse events including drug-induced bone-marrow toxicity, liver injury, pancreatitis and GI bleed, [9, 10] these were not seen in our case series.

Several observations deserve further elaboration. Both patients (patients 1 and 2) with good outcomes were previously healthy young males and received TCZ early in the course of their illness prior to need for invasive or noninvasive mechanical ventilation. This is consistent with reports from multiple large studies indicating better outcomes in younger patients with COVID-19 infection [3, 4, 10, 11]. Also, IL-6 levels were lower in these two patients compared to the others who had poor response. Patient 5 also received TCZ prior to endotracheal intubation and mechanical ventilation, however she was 73 years old and had comorbidities hypertension, gout and atrial fibrillation. Of the remaining four patients, all had severe COVID-19 infection, three were intubated on day 1 of hospitalization, one patient was intubated on hospital day 3. TCZ did not show benefit in these patients and 2 of them died after a prolonged and complicated hospital stay. While it is unknown if these patients may have benefited from a second dose of TCZ, our experience adds to the accumulating data regarding the limited benefit of single dose TCZ in patients with severe advanced COVID-19 disease. Also, 4 out of the 7 patients subsequently developed bacterial pneumonias. We do not know the true relationship of pneumonia with TCZ therapy in these patients, infections are well known complications associated with TCZ use in other diseases [7]. The exact role of IL-6 in the pathogenesis of COVID-19 associated pneumonia needs to be further elucidated to better understand the role of TCZ in the management of this disease. IL-6 increases after TCZ dose and it is not yet understood if this may exacerbate the inflammatory response or whether it is a transient and benign phenomenon.

Initial reports had suggested good results of TCZ therapy in patients with severe COVID-19 infection $[10,11]$. However, after results from larger clinical studies, the enthusiasm for TCZ was tempered by lack of survival benefit $[12,13]$.

Early in the course of the pandemic, healthcare bodies had advised against use of corticosteroids in patients with COVID-19 infection. However, it is now considered the standard of care in management of hospitalized patients requiring supplemental oxygen therapy. Newer studies have shown a trend towards better outcome in patients treated with combination TCZ and corticosteroids [14, 15]. The concomitant use of corticosteroids may result in ameliorating the effect of the transient elevation of IL- 6 after TCZ dose and this may be a possible explanation for better outcomes when TCZ and corticosteroids are used together.

\section{Conclusions}

Tocilizumab benefitted two young male patients who received a single dose prior to need for invasive ventilation. Benefit was not seen in 5 older individuals with advanced disease. IL-6 was elevated in these patients and increased even further after the dose of Tocilizumab. It remains unknown if these 5 patients may have benefited from a second dose of TCZ. Our experience adds to the accumulating data regarding the limited benefit of single dose TCZ in patients with severe advanced COVID-19 disease. Hence the exact dose, number of doses and optimal time of administration of TCZ during the course of the disease, as well as benefits when employed along with other agents like corticosteroids, remdesivir and convalescent plasma needs to be evaluated further. Meanwhile as we await - with guarded optimism, the results of clinical trials involving a larger number of patients, as well as definitive conclusions regarding use of tocilizumab and corticosteroids in combination, we 
hope that this short case series adds to the growing information regarding use of Tocilizumab in the management of patients with COVID-19 infection.

\begin{tabular}{|c|c|c|c|c|c|c|c|c|c|c|}
\hline No. & Sex & $\begin{array}{l}A \quad g \quad e \\
\text { (years) }\end{array}$ & $\begin{array}{l}\text { Day of } \mathrm{TC} \\
\mathrm{t} \mathrm{h} \text { e } \mathrm{r} \text { a } \mathrm{p} y \\
\text { from ICU } \\
\text { admission }\end{array}$ & $\begin{array}{ll}\text { Symptoms } \\
\text { presentation }\end{array}$ & Comorbidities & $\begin{array}{l}\text { Weight } \\
(\mathrm{kg})\end{array}$ & CRP & Ferritin & D-dimer & Il-6 \\
\hline 1 & M & 28 & 2 & $\begin{array}{lr}\text { Fever, } & \text { shortness } \\
\text { of } & \text { breath, } \\
\text { nonproductive cough }\end{array}$ & nil & 130 & 239.9 & 1569 & 1.92 & 47 \\
\hline 2 & M & 48 & 5 & $\begin{array}{lr}\text { Fever, } & \text { shortness } \\
\text { of } & \text { breath, } \\
\text { nonproductive cough }\end{array}$ & nil & 81.6 & 148 & 1736 & 0.59 & 64.6 \\
\hline 3 & M & 58 & 4 & $\begin{array}{lr}\text { Fever, } & \text { shortness } \\
\text { of } & \text { breath, } \\
\text { nonproductive cough }\end{array}$ & $\begin{array}{l}\text { Hydrocephalus, } \\
\text { V-P shunt }\end{array}$ & 78 & 203.76 & 767 & 17.37 & 625.9 \\
\hline 4 & $\mathrm{~F}$ & 61 & 10 & $\begin{array}{lr}\text { Fever, } & \text { shortness } \\
\text { of } & \text { breath, } \\
\text { nonproductive cough }\end{array}$ & $\begin{array}{l}\text { Hypertension, } \\
\text { fatty liver }\end{array}$ & 74 & 147.4 & 3144 & 5.24 & 53.5 \\
\hline 5 & $\mathrm{~F}$ & 73 & 3 & $\begin{array}{l}\text { Fever, shortness of } \\
\text { breath }\end{array}$ & $\begin{array}{l}\text { Hypertension, } \\
\text { gout, atrial } \\
\text { fibrillation }\end{array}$ & 118 & 241.37 & 624 & 0.86 & 238.1 \\
\hline 6 & M & 61 & 2 & $\begin{array}{l}\text { Fever, shortness of } \\
\text { breath }\end{array}$ & $\begin{array}{l}\text { Hypertension, } \\
\text { hyperlipidemia, } \\
\text { d i a b e t e s, } \\
\text { d e pres s i on, } \\
\text { cardiomyopathy }\end{array}$ & 113 & 191.25 & 948 & 2.35 & no \\
\hline 7 & $\mathrm{~F}$ & 57 & 4 & $\begin{array}{l}\text { Fever, shortness of } \\
\text { breath }\end{array}$ & $\begin{array}{l}\text { Hypertension, } \\
\text { diabetes }\end{array}$ & 144 & 134.2 & 808 & 1.42 & 2481.3 \\
\hline
\end{tabular}

Table 1: Clinical and laboratory characteristics of patients receiving Tocilizumab

\begin{tabular}{|l|l|l|}
\hline No. & Sputum Culture & Antibiotics (days) \\
\hline 1 & Poor quality & CTX (7), Azi (2) \\
\hline 2 & Respiratory flora & CTX (7), Doxycycline (5) \\
\hline 3 & Respiratory flora & CTX (10), Azi (5) \\
\hline 4 & Respiratory flora & CTX (7), Doxycycline (6) \\
\hline 5 & Nil & $\begin{array}{l}\text { CTX (2), Doxycycline (4), Azi (2), } \\
\text { Linezolid (3) }\end{array}$ \\
\hline 6 & Nil & CTX (1), Azi (1) \\
\hline 7 & Negative & CTX (5), Azi (3) \\
\hline
\end{tabular}

Table 2: Sputum culture results and antibiotic use in patients prior to receiving Tocilizumab

$$
(\mathrm{CTX}=\text { ceftriaxone, } \mathrm{Azi}=\text { azithromycin })
$$


References

1. Su S, Wong G, Shi W et al., (2016). Epidemiology, genetic recombination, and pathogenesis of Coronaviruses. Trends Microbiol, 24(6): 490-502.

2. Gulati A, Pomeranz C, Qamar Z et al., (2020). A comprehensive review of manifestations of novel Coronaviruses in the context of deadly COVID-19 global pandemic. Am J Med Sci, 360(1): 5-34.

3. Richardson S, Hirsch JS, Narasimhan M et al., (2020). Presenting characteristics, comorbidities, and outcomes among 5700 patients hospitalized with COVID-19 in the New York City area, 26; 323(20): 2052-2059.

4. Grasselli G, Greco M, Zanella A et al., (2020). Risk factors associated with mortality among patients with COVID-19 in intensive care units in Lombardi, Italy. JAMA Intern Med, 180 (10): 1345-1355.

5. Channappanavar R, Perlman S.Semin (2017). Pathogenic human coronavirus infections: causes and consequences of cytokine storm and immunopathology. Immunopathol, 39(5): 529-539.

6. Jamilloux Y, Henry T, Belot A, et al., (2020). Should we stimulate or suppress immune responses in COVID-19? Cytokine and anti-cytokine interventions. Autoimmun Rev, 19(7): 102567.

7. Burmester GR, Rubbert-Roth A, Cantagrel A, et al., (2016). Efficacy and safety of subcutaneous tocilizumab versus intravenous tocilizumab in combination with traditional DMARDs in patients with RA at week 97 (SUMMACTA). Ann Rheum Dis, 75(1): 68-74.

8. https://www.cancernetwork.com/view/fda-approvesphase-iii-clinical-trial-tocilizumab-covid-19-pneumonia. Accessed 3/10/2021.

9. Gatti M, Fusaroli M, Caraceni P, et al., (2020). Serious adverse events with tocilizumab: Pharmacovigilance as an aid to prioritize monitoring in COVID-19. Br J Clin Pharmacol.

10. Toniati P, Piva S, Cattalini M, et al., (2020). Tocilizumab for the treatment of severe COVID-19 pneumonia with hyperinflammatory syndrome and acute respiratory failure: A single center study of 100 patients in Brescia, Italy. Autoimmun Rev. 19(7): 102568.

11. Zakowski P, Tran HP, et al., (2021). Compassionate Use of Tocilizumab for Treatment of SARS-CoV-2 Pneumonia. Jordan SC. N Engl J Med, 384(1):20-30.

12. Salama C, Han J, Yau L, et al., (2021). Tocilizumab in patients hospitalized with COVID-19 pneumonia. $N$ Engl J Med, 384(1): 20-30.

13. ww w.roche.com/investors/updates/invupdate-2020-07-29.htm. Accessed 2/25/21.
14. Mikulska M, Nicolini LA, Signori A, et al., (2020). Tocilizumab and steroid treatment in patients with COVID-19 pneumonia. PLoS One, 15(8): e 0237831.

15. Narain S, Stefanov DG, Chau AS, et al., (2020). Comparative Survival Analysis of Immunomodulatory Therapy for Coronavirus Disease 2019 Cytokine Storm. Chest, 159(3): 933-948.
Copyright: (C2021 Alwyn Rapose. This is an open-access article distributed under the terms of the Creative Commons Attribution License, which permits unrestricted use, distribution, and reproduction in anymedium, provided the original author and source are credited. 